\title{
High concentrations of pepsin in bronchoalveolar lavage fluid from children with cystic fibrosis are associated with high interleukin-8 concentrations
}

\author{
P McNally, ${ }^{1,2}$ E Ervine, ${ }^{3}$ M D Shields, ${ }^{3}$ B D Dimitrov, ${ }^{4}$ B El Nazir, ${ }^{2}$ C C Taggart, ${ }^{1}$ \\ C M Greene, ${ }^{1}$ N G McElvaney, ${ }^{1}$ P Greally ${ }^{2}$
}

- An additional appendix 1 is published online only. To view this file please visit the journa online (http://thorax.bmj.com).

${ }^{1}$ Respiratory Research Division, Royal College of Surgeons in Ireland, Beaumont Hospital, Dublin, Ireland

2Department of Paediatric

Respiratory Medicine, National Children's Hospital, Dublin, Ireland

${ }^{3}$ Respiratory Research Cluster, Centre for Infection and Immunity, Queen's University Belfast, Belfast, UK

${ }^{4}$ Department of General Practice, Royal College of Surgeons in Ireland, Dublin, Ireland

\section{Correspondence to}

Dr Paul McNally, Department of Respiratory Medicine, Our Lady's Children's Hospital, Crumlin, Dublin 12, Ireland; paul.mcnally@olchc.ie

Received 30 November 2009 Accepted 22 October 2010 Published Online First 15 December 2010

\begin{abstract}
Background Gastro-oesophageal reflux is common in children with cystic fibrosis (CF) and is thought to be associated with pulmonary aspiration of gastric contents. The measurement of pepsin in bronchoalveolar lavage (BAL) fluid has recently been suggested to be a reliable indicator of aspiration. The prevalence of pulmonary aspiration in a group of children with CF was assessed and its association with lung inflammation investigated. Methods This was a cross-sectional case-control study. BAL fluid was collected from individuals with CF $(n=31)$ and healthy controls $(n=7)$. Interleukin-8 (IL-8), pepsin, neutrophil numbers and neutrophil elastase activity levels were measured in all samples. Clinical, microbiological and lung function data were collected from medical notes.
\end{abstract}

Results The pepsin concentration in BAL fluid was higher in the CF group than in controls (mean (SD) 24.4 (27.4) $\mathrm{ng} / \mathrm{ml}$ vs $4.3(4.0) \mathrm{ng} / \mathrm{ml}, \mathrm{p}=0.03)$. Those with CF who had raised pepsin concentrations had higher levels of IL-8 in the BAL fluid than those with a concentration comparable to controls $(3.7(2.7) \mathrm{ng} / \mathrm{ml}$ vs $1.4(0.9) \mathrm{ng} / \mathrm{ml}, \mathrm{p}=0.004)$. Within the CF group there was a moderate positive correlation between pepsin concentration and IL-8 in BAL fluid ( $r=0.48, p=0.04)$. There was no association between BAL fluid pepsin concentrations and age, sex, body mass index z score, forced expiratory volume in $1 \mathrm{~s}$ or Pseudomonas aeruginosa colonisation status.

Conclusions Many children with CF have increased levels of pepsin in the BAL fluid compared with normal controls. Increased pepsin levels were associated with higher IL-8 concentrations in BAL fluid. These data suggest that aspiration of gastric contents occurs in a subset of patients with CF and is associated with more pronounced lung inflammation.

\section{BACKGROUND}

The symptoms of gastro-oesophageal reflux (GOR) are very common in children with cystic fibrosis. ${ }^{1}$ Studies using oesophageal manometry and $\mathrm{pH}$ probes have demonstrated high levels of GOR in both children and adults with cystic fibrosis. Scott et al found GOR in $20 \%$ of those aged $<5$ years of age while Ledson et al found a prevalence of $80 \%$ in adult patients, some of whom had tracheal acidification in addition, suggesting micro-aspiration. ${ }^{1-3}$ According to Blondeau et al, GOR is common in adults with CF and is associated with increased cough and poorer lung function. ${ }^{4}$ Several patients in that study had bile acids in their bronchoalveolar lavage (BAL) fluid, suggesting a link with aspiration. A study by Button et al found that GOR was present in $90 \%$ of severely affected adult patients with CF before transplantation and $84 \%$ after transplantation. ${ }^{5}$

The diagnosis and treatment of GOR is a common component of care at most CF centres. Many patients report symptom improvement with gastric acid suppressive therapy. However, treatment with acid blockade will not prevent non-acid reflux or its aspiration. ${ }^{6}$ The detection and treatment of pulmonary aspiration is fraught with difficulty. The diagnosis is often made with a combination of clinical, laboratory and radiological tests. However, the overall sensitivity and specificity of these tests alone or in combination remains poor. An ideal test for aspiration would involve the detection of a marker within the lungs that clearly should not be present within the tracheobronchial tree under normal conditions. The marker ought to be easily recovered and reliably detectable for a period of time after acute aspiration. Recently, several investigators have focused on pepsin, a digestive protease that degrades food proteins into peptides. It is expressed as a pro-form zymogen, pepsinogen, which is released by chief cells in the stomach and subsequently activated by hydrochloric acid cleaving it to the active form, pepsin. A link between pulmonary aspiration and the detection of pepsin in tracheal aspirates or BAL fluid has been demonstrated in human and animal studies. ${ }^{7-16}$ Farrell et al recently employed the pepsin ELISA used in this study to look specifically at aspiration of gastric contents in children without $\mathrm{CF}^{10}$

Given the increased prevalence of GOR in children with $\mathrm{CF}^{1-4}$ and the link between aspiration of gastric contents and lung inflammation, ${ }^{17}$ we sought to determine whether pulmonary aspiration was occurring in children with CF and, if so, whether this was associated with more marked pulmonary inflammation.

\section{METHODS \\ Patient selection}

We studied a consecutive group of children with CF referred for bronchoscopy during the period July 2005 to July 2007 who satisfied the selection criteria and provided written informed consent. There was a range of different indications for these procedures: for routine microbiological surveillance in non-sputum producers, to confirm or follow-up a growth of Pseudomonas aeruginosa in a throat 
swab, to investigate ongoing respiratory symptoms out of context with culture results and, in some cases, opportunistically at the time of other elective procedures in these patients. Inclusion criteria were age $0-17$ years, an abnormal sweat test and the presence of at least one CF mutation. Exclusion criteria were the presence of an acute CF exacerbation, a diagnosis of allergic bronchopulmonary aspergillosis, treatment with systemic steroids and a clinical diagnosis of asthma. Non-CF healthy control samples were obtained from children without lung disease undergoing elective non-pulmonary surgical procedures. Inclusion criteria were age $0-17$ years. Exclusion criteria were any history of recurrent respiratory symptoms, respiratory symptoms on the day of surgery or a diagnosis of asthma.

\section{Bronchoalveolar lavage fluid}

Bronchoscopy was performed via a laryngeal mask airway to avoid contamination from the upper airway and aspiration of gastric contents during the procedure. Bronchoalveolar lavage was performed in the lingula and right middle lobe by instilling $1 \mathrm{ml} / \mathrm{kg}$ sterile normal saline per lobe. Return was typically in the region of $40 \%$. Specimens from right and left lungs were pooled. BAL fluid was centrifuged at $2000 \times \mathrm{g}$ for $10 \mathrm{~min}$ and the supernatant aliquoted and stored at $-80^{\circ} \mathrm{C}$

\section{Pepsin ELISA}

Pepsin concentrations were measured in clinical samples by an in-house ELISA assay with antiporcine pepsin antibodies developed at Queen's University Belfast, as previously described ${ }^{10}$ (see appendix 1 in online supplement).

\section{IL-8 ELISA}

IL-8 was measured by ELISA using 1:125 mouse anti-IL-8 as primary antibody and 1:2500 biotinylated goat anti-IL-8 as detection antibody (all R\&D systems, Abingdon, Oxon, UK).

\section{Study design and sample size estimation}

This was a cross-sectional case-control study. All relevant demographic, clinical and laboratory data were collected from the patients' medical notes and recorded in a dedicated case record form. There were limited data to inform sample size calculation, however estimates were calculated using the Satterthwaite t test of equal means (unequal variances, unequal groups). Presuming a minimum average pepsin concentration of $24 \mathrm{ng} / \mathrm{ml}$ in cases (based on pepsin values in children with aspiration $)^{10}$ and a maximum average pepsin concentration of $<5 \mathrm{ng} / \mathrm{ml}$ in control subjects, with a ratio of cases to controls of $5: 1$ and at two-tailed $\mathrm{p}<0.05$ (power $>85 \%$ ), a minimum of 27 cases and 5 controls were required.

\section{Statistical analyses}

Descriptive statistics and percentile methods were used. The normality of the distribution of continuous variables was assessed by the Kolmogorov-Smirnov test. The groups and subgroups were compared by two-tailed independent sample Student $t$ test when normally distributed or by the nonparamtric Mann-Whitney $U$ test, as appropriate. The associations between different variables among children with CF were evaluated by univariate analyses. Pearson correlation coefficient was used for establishing correlations between normally distributed continuous variables or, alternatively, the Spearman rho coefficient was used. Continuous data are expressed as mean (SD) unless otherwise specified. The statistical significance of all tests was assumed at two-tailed $p<0.05$. All analyses were performed with GraphPad Prism software.
Table 1 Baseline characteristics and mean bronchoalveolar lavge fluid pepsin values of the two groups

\begin{tabular}{llll}
\hline & Cases $(\mathbf{n}=\mathbf{3 1})$ & Controls $(\mathbf{n}=\mathbf{7})$ & $\mathbf{p}$ Value \\
\hline Mean (range) age (years) & $10.4(0.4-17.7)$ & $8.3(3.7-15.1)$ & 0.51 \\
Gender (M/F) & $20 / 11$ & $3 / 4$ & 0.30 \\
Mean \pm SD pepsin levels $(\mathrm{ng} / \mathrm{ml})$ & $24.4 \pm 27.4$ & $4.3 \pm 4$ & 0.03 \\
\hline
\end{tabular}

Differences in individual means were calculated by Student $t$ test for continuous data and $\chi 2$ for categorical data.

\section{RESULTS}

\section{Study patients}

Overall, 31 cases with CF and 15 controls were identified as eligible participants (table 1). After identification, only eight controls did not provide written informed consent and did not participate and complete the study, so a total of 38 subjects were recruited and analysed ( $n=31$ cases, mean age 10.4 years (range 0.4-17.7); $\mathrm{n}=7$ controls, mean age 8.3 years (range $3.7-15.1$ )).

\section{Clinical characteristics}

There was no significant difference in age or gender distribution, $P$ aeruginosa colonisation status or previous investigations for GOR between the two groups of individuals with CF (CF with high vs CF with low BAL fluid pepsin concentration, table 2).

\section{BAL fluid pepsin level is raised in individuals with CF}

Mean pepsin levels in the CF group (24.4 (27.4) ng/ml) were higher than those in the control group $(4.3$ (4.0) $\mathrm{ng} / \mathrm{ml}, \mathrm{p}=0.03$, figure 1). It should be noted that some pepsin was detected in most samples, even those from controls. A large proportion $(12 / 31)$ of the CF group had pepsin levels within the same range as the control group. As a low concentration of pepsin was detectable in BAL fluid from controls, the pepsin concentration in those with CF was considered 'high' if it was 295 th percentile for controls $(10.4 \mathrm{ng} / \mathrm{ml})$ and 'low' if it was below this value.

BAL fluid pepsin levels correlate with IL-8 in individuals with CF We sought to investigate whether this increase in pepsin values was associated with increased evidence of inflammation. BAL fluid IL-8 levels were significantly higher in those with high pepsin levels $(3.7(2.7) \mathrm{ng} / \mathrm{ml})$ than in those with low pepsin (1.4 (0.9) ng/ml, $\mathrm{p}=0.004$, figure 2). Furthermore, there was a positive correlation between BAL fluid levels of pepsin and of IL-8 in the group with CF $(r=0.48, n=31$, figure 3$)$. Increased

Table 2 Summary of clinical characteristics, test results and BAL fluid inflammatory indices between patients with cystic fibrosis with high pepsin levels and those with low levels

\begin{tabular}{llll}
\hline & $\begin{array}{l}\text { High pepsin } \\
(\mathbf{n = 1 9 )}\end{array}$ & $\begin{array}{l}\text { Low pepsin } \\
(\mathbf{n = 1 2 )}\end{array}$ & p Value \\
\hline Mean age (years) & 10.73 & 10.07 & 0.79 \\
Gender (M/F) & $13 / 6$ & $7 / 5$ & 0.53 \\
Neutrophils $\left(\times 10^{6} / \mathrm{ml}\right.$ BAL) & 5.52 & 2.61 & 0.15 \\
BMI z score & -0.94 & -0.7 & 0.66 \\
FEV 1 (\% predicted) & 63 & 68.4 & 0.73 \\
BAL NE activity (nM) & 56 & 37 & 0.29 \\
PPI use & $6 / 19(32 \%)$ & $3 / 12(25 \%)$ & 0.69 \\
pH probe performed & $5 / 19(26 \%)$ & $1 / 12(8 \%)$ & 0.21 \\
Positive pH probe & $5 / 5(100 \%)$ & $1 / 1(100 \%)$ & NS \\
Chronic $P$ aeruginosa colonisation & $6 / 19(32 \%)$ & $3 / 12(25 \%)$ & 0.69 \\
\hline
\end{tabular}

Differences in individual means were calculated by Student $\mathrm{t}$ test or Mann-Whitney $\mathrm{U}$ test for continuous data, and $\chi^{2}$ test for categorical data.

$\mathrm{BAL}$, bronchoalveolar lavage; $\mathrm{BMI}$, body mass index; $\mathrm{FEV}_{1}$, forced expiratory volume in $1 \mathrm{~s}$; $\mathrm{NE}$, neutrophil elastase; NS, not significant; PPI, proton pump inhibitor. 


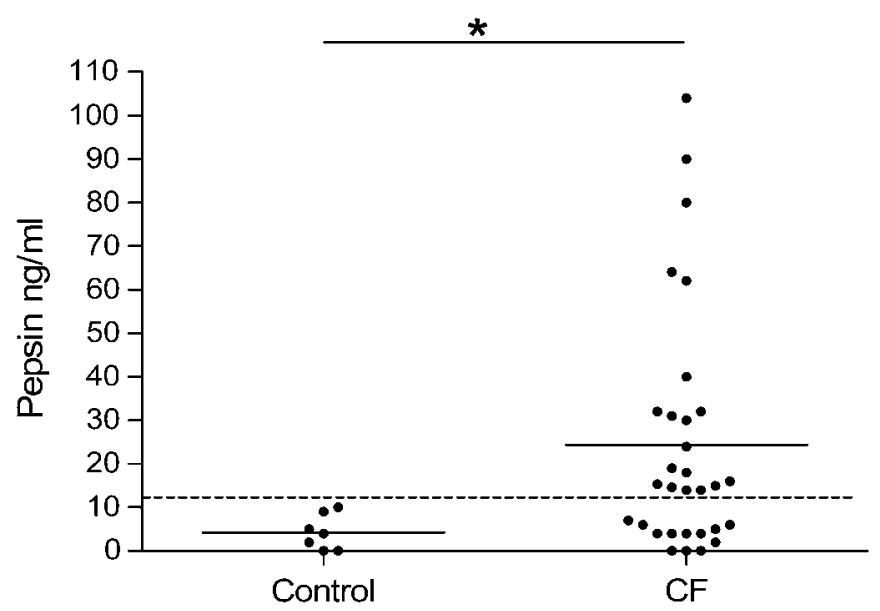

Figure 1 Pepsin levels in bronchoalveolar lavage (BAL) fluid from controls and patients with cystic fibrosis (CF). The dotted line (10.4 ng/ $\mathrm{ml}$ ) represents the 95th percentile value in the controls. Differences in individual means were assessed by the Student $t$ test.

neutrophil counts in BAL fluid were observed in the CF group with high BAL fluid pepsin concentrations compared with those with low BAL fluid pepsin concentrations (5.5 (6.9) vs $2.6(3.8) \times$ $10^{6}$ cells $\left./ \mathrm{ml}\right)$, although the difference was not statistically significant ( $p=0.15$, t test).

\section{DISCUSSION}

In this study of children with CF undergoing bronchoscopy as part of their clinical care, we found that, in more than half, BAL fluid pepsin concentrations were raised, in keeping with pulmonary aspiration. Moreover, those with higher BAL fluid pepsin concentrations also had higher IL-8 concentrations, suggesting that ongoing pulmonary aspiration is contributing to airways inflammation.

The diagnosis of pulmonary aspiration remains a challenge. No single test to date has been found to be $100 \%$ sensitive or specific, and the diagnosis is often based on clinical suspicion with some supporting evidence from radiological, laboratory and physiological tests. To date, the most widely used method for detection of pulmonary aspiration has been microscopic analysis

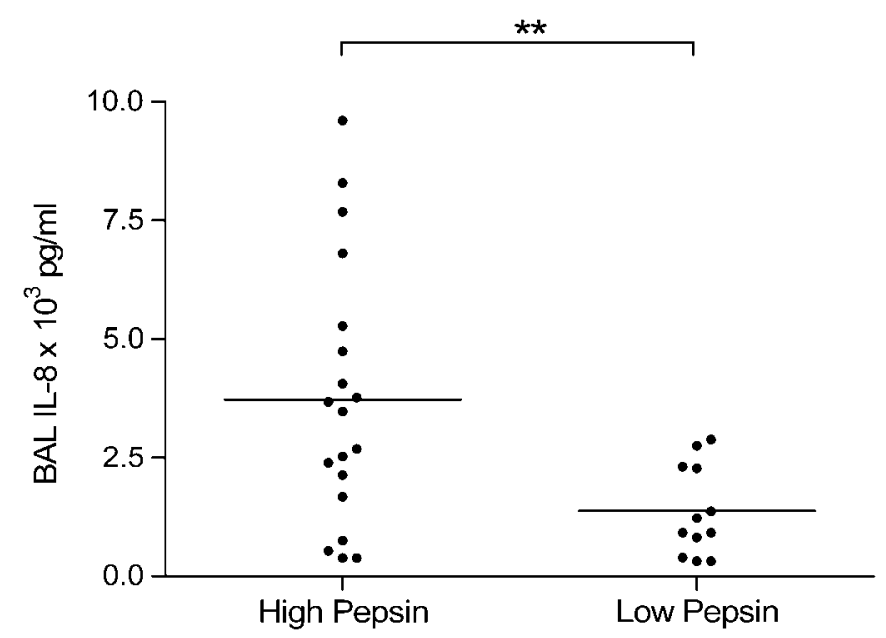

Figure 2 Bronchoalveolar lavage (BAL) fluid levels of interleukin 8 (IL-8) in patients with cystic fibrosis with high and low BAL fluid pepsin values. Differences in means were analysed by the Student $t$ test.

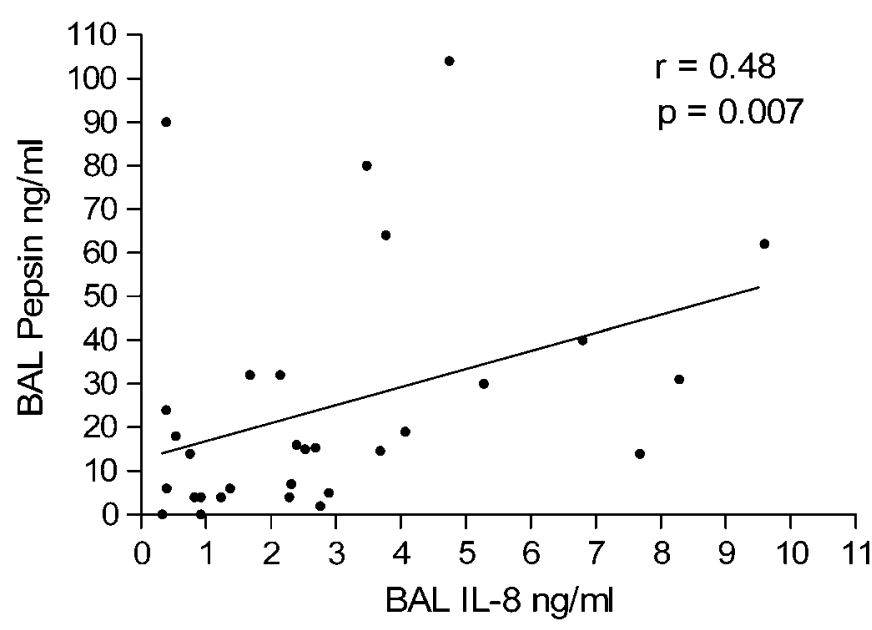

Figure 3 Correlation between pepsin level and interleukin 8 (IL-8) in bronchoalveolar lavage (BAL) fluid in patients with cystic fibrosis. Correlation was determined by the Pearson correlation coefficient.

of secretions for the presence of lipid-laden macrophages. An index comparing the proportion of lipid-laden to normal macrophages must be calculated and used for comparison-the lipid-laden macrophage index (LLMI). This test is labour-intensive, operator-dependent and has poor specificity and variable sensitivity. ${ }^{18} 19$ No clear reference standards exist for this method. The technique has been evaluated in children with CF. Kazachkov et al found a raised LLMI in children with CF and with other chronic pulmonary conditions, but no normal controls were assessed. The LLMI was higher in the CF group, but this did not correlate with inflammatory markers in BAL fluid or symptoms suggestive of GOR in either group. ${ }^{20}$

In situations where aspiration of gastric refluxate into the lungs is strongly suspected, the definitive treatment usually involves surgical fundoplication. However, surgical procedures in CF, especially those which may alter physiological function, are not undertaken lightly and robust evidence for their need should be obtained. The measurement of pepsin levels in BAL fluid has the potential to provide such confirmatory documentation. Pepsin assays have the potential to significantly improve the sensitivity and specificity of the current diagnostic approach to aspiration in the clinical setting. The pepsin assay used in this study was specifically developed and validated for detection of pulmonary aspiration in children. ${ }^{10}$

The fact that pepsin is produced in the gastrointestinal tract and not the lung fulfils one of the most important requirements of a reliable marker of aspiration. However, a study in healthy young adults has shown that a significant portion of patients aspirate nasopharyngeal secretions during sleep. ${ }^{21}$ In patients with GOR, in whom the upper oesophageal sphincter tone drops during deep sleep, the potential exists for some microaspiration of gastric contents. ${ }^{22}$ Thus, the presence per se of pepsin at any level in the BAL fluid may not necessarily always be an abnormal finding. In this context, the detection of low levels of pepsin in the BAL fluid of some of our control patients is perhaps not surprising. Several of the individuals with CF have similarly low levels of pepsin, however many more had levels that were significantly higher than control values, perhaps indicating more significant degrees of aspiration. A clear segregation between low and high pepsin levels can be observed using the 95th percentile cut-off (figure 2). There are no published data for the concentration of pepsin in the BAL fluid that may be seen in the absence of respiratory symptoms or pulmonary 
inflammation, so deciding which levels are potentially physiological and which are clearly pathological presents a challenge. Individuals in the control group were completely asymptomatic from a respiratory perspective at the time of bronchoscopy, and had normal differential cell counts and negligible levels of inflammatory cytokines in the BAL fluid (data not shown). We therefore feel confident that the pepsin levels measured in this group are unlikely to be clinically significant and represent a true normal range. The presence of low levels of pepsin in otherwise healthy children is surprising but may reflect microaspiration during sleep, as has been shown in adults. Little is known about how long pepsin may be detectable in the lungs after aspiration of gastric contents.

The finding of increased levels of IL-8 among the group with the higher pepsin levels and the correlation between levels of IL-8 and pepsin in BAL fluid suggests that high pepsin levels are associated with more severe lower airway inflammation. Similar findings have been observed in lung transplant recipients. ${ }^{16} 23$ This is the first time that a link has been demonstrated between a biological marker of aspiration and inflammation in relatively stable individuals with CF (mean forced expiratory volume in $1 \mathrm{~s}$ $66 \%$ ). This association is strengthened by the fact that both measurements are derived from the same sample from any given patient, minimising the effect of even small variations in BAL fluid concentration between patients. Our finding of the link between pulmonary aspiration and inflammation in CF is potentially an extremely important discovery for individuals with CF. Indeed, the relevance is amplified when one considers that many of the children in the study had no symptoms of GOR. It therefore seems likely that silent aspiration of gastric contents may be a more common problem than most CF clinicians would anticipate.

Contrary to what one might expect, we found no increase in pepsin levels in BAL fluid with age, nor did we see an association between pepsin levels in BAL fluid and lung function. This may be the result of the small numbers and the cross-sectional nature of the study. It is likely that the degree of aspiration in patients varies over time, ${ }^{21}$ so a longitudinal study may be better equipped to address these associations.

This study demonstrates for the first time that aspiration of gastric contents occurs in a subset of clinically stable patients with CF and is associated with more pronounced lung inflammation. Further longitudinal studies are required to determine the long-term clinical significance of this observation.

\section{Competing interests None.}

Ethics approval This study was conducted with the approval of the St James's Hospital/Adelaide and Meath Hospital incorporating the National Children's Hospital, Dublin, Ireland and full informed parental consent was obtained for all procedures.

Provenance and peer review Not commissioned; externally peer reviewed.

\section{REFERENCES}

1. Scott RB, O'Loughlin EV, Gall DG. Gastroesophageal reflux in patients with cystic fibrosis. J Pediatr 1985;106:223-7.

2. Ledson MJ, Tran J, Walshaw MJ. Prevalence and mechanisms of gastrooesophageal reflux in adult cystic fibrosis patients. J $R$ Soc Med 1998;91:7-9.

3. Ledson MJ, Wilson GE, Tran J, et al. Tracheal microaspiration in adult cystic fibrosis. J $R$ Soc Med 1998;91:10-12.

4. Blondeau K, Dupont LJ, Mertens V, et al. Gastro-oesophageal reflux and aspiration of gastric contents in adult patients with cystic fibrosis. Gut 2008; 57:1049-55.

5. Button BM, Roberts S, Kotsimbos TC, et al. Gastroesophageal reflux (symptomatic and silent): a potentially significant problem in patients with cystic fibrosis before and after lung transplantation. J Heart Lung Transplant 2005;24:1522-9.

6. Blondeau K, Mertens V, Vanaudenaerde BA, et al. Gastro-oesophageal reflux and gastric aspiration in lung transplant patients with or without chronic rejection. Eur Respir J 2008;31:707-13.

7. Farhath S, Aghai ZH, Nakhla T, et al. Pepsin, a reliable marker of gastric aspiration, is frequently detected in tracheal aspirates from premature ventilated neonates: relationship with feeding and methylxanthine therapy. J Pediatr Gastroenterol Nutr 2006;43:336-41

8. Metheny NA, Clouse RE, Chang YH, et al. Tracheobronchial aspiration of gastric contents in critically ill tube-fed patients: frequency, outcomes, and risk factors. Crit Care Med 2006;34:1007-15.

9. Metheny NA, Dahms TE, Chang YH, et al. Detection of pepsin in tracheal secretions after forced small-volume aspirations of gastric juice. JPEN J Parenter Enteral Nutr 2004;28:79-84.

10. Farrell S, McMaster C, Gibson D, et al. Pepsin in bronchoalveolar lavage fluid: a specific and sensitive method of diagnosing gastro-oesophageal reflux-related pulmonary aspiration. J Pediatr Surg 2006;41:289-93.

11. Ward C, Forrest IA, Brownlee IA, et al. Pepsin like activity in bronchoalveolar lavage fluid is suggestive of gastric aspiration in lung allografts. Thorax 2005:60:872-4.

12. Ufberg JW, Bushra JS, Patel D, et al. A new pepsin assay to detect pulmonary aspiration of gastric contents among newly intubated patients. Am J Emerg Med 2004:22:612-14

13. Tasker A, Dettmar PW, Panetti M, et al. Reflux of gastric juice and glue ear in children. Lancet 2002;359:493.

14. Tasker A, Dettmar PW, Panetti M, et al. Is gastric reflux a cause of otitis media with effusion in children? Laryngoscope 2002;112:1930-4.

15. Starosta V, Kitz R, Hartl D, et al. Bronchoalveolar pepsin, bile acids, oxidation, and inflammation in children with gastroesophageal reflux disease. Chest 2007; 132:1557-64

16. Stovold R, Forrest IA, Corris PA, et al. Pepsin, a biomarker of gastric aspiration in lung allografts: a putative association with rejection. Am J Respir Crit Care Med 2007:175:1298-303.

17. Bregeon F, Papazian L, Delpierre S, et al. Role of proinflammatory activity contained in gastric juice from intensive care unit patients to induce lung injury in a rabbit aspiration model. Crit Care Med 2008;36:3205-12.

18. Furuya ME, Moreno-Cordova V, Ramirez-Figueroa JL, et al. Cutoff value of lipid-laden alveolar macrophages for diagnosing aspiration in infants and children. Pediatr Pulmonol 2007:42:452-7.

19. Knauer-Fischer S, Ratjen F. Lipid-laden macrophages in bronchoalveolar lavage fluid as a marker for pulmonary aspiration. Pediatr Pulmonol 1999;27:419-22.

20. Kazachkov MY, Muhlebach MS, Livasy CA, et al. Lipid-laden macrophage index and inflammation in bronchoalveolar lavage fluids in children. Eur Respir J 2001;18:790-5.

21. Gleeson K, Eggli DF, Maxwell SL. Quantitative aspiration during sleep in normal subjects. Chest 1997;111:1266-72.

22. Kahrilas PJ, Dodds WJ, Dent J, et al. Effect of sleep, spontaneous gastroesophageal reflux, and a meal on upper esophageal sphincter pressure in normal human volunteers. Gastroenterology 1987;92:466-71.

23. D'Ovidio F, Singer LG, Hadjiliadis D, et al. Prevalence of gastroesophageal reflux in end-stage lung disease candidates for lung transplant. Ann Thorac Surg 2005;80:1254-60. 